\title{
Thalamo-hippocampal pathway regulates incidental memory load: insights from sex differences
}

\author{
Authors: G. Torromino ${ }^{1,2 * \dagger} \dagger$, V. Loffredo ${ }^{1,2 *}$, D. Cavezza $^{2}$, F. Esposito ${ }^{1}$, A. H. Crevenna ${ }^{3}$, M. Gioffrè ${ }^{4}$, \\ M. De Risi ${ }^{1,2}$, A. Treves ${ }^{5}$, M. Griguoli ${ }^{6,7}$, E. De Leonibus ${ }^{1,2} \#$
}

\author{
Affiliations: \\ ${ }^{1}$ Telethon Institute of Genetics and Medicine, Telethon Foundation, Pozzuoli (Naples), Italy. \\ ${ }^{2}$ Institute of Biochemistry and Cell Biology (IBBC), National Research Council, Monterotondo (Rome), Italy. \\ ${ }^{3}$ Neurobiology and Epigenetics Unit, European Molecular Biology Laboratory (EMBL), Monterotondo (Rome), \\ Italy. \\ ${ }^{4}$ Institute for Microelectronics and Microsystems (IMM), National Research Council, Naples, Italy. \\ ${ }^{5}$ SISSA - Cognitive Neuroscience, Trieste, Italy. \\ ${ }^{6}$ European Brain Research Institute (EBRI), Rome, Italy. \\ ${ }^{7}$ Institute of Neuroscience (IN), National Research Council, Pisa, Italy.
}

\#Correspondence to: deleonibus@tigem.it

* These authors contributed equally to this work

\begin{abstract}
Memory can be challenged by increasing both its required duration and the amount of information to be encoded, namely the memory load. The dorsal hippocampus (dHP) has been involved in memory consolidation, which is the stabilization of a trace from short-term (STM) to long-term memory (LTM), as well as in the ability to process high information load. However, how memory load influences memory consolidation, and the underlying neural mechanisms, are yet unknown.

To address this question, we used male and female mice that, despite having in our Different Object recognition Task (DOT) the same STM capacity of 6 objects, spontaneously show differences in the number of objects directly transferred to LTM, when tested over longer delays. Males memorize all 6 objects encoded, while females remember only up to 4 , both at 1 and $24 \mathrm{~h}$ delays. Interestingly, males activate more the dHP (as measured by c-Fos expression), while females the thalamic nucleus reuniens (RE). Optogenetic inhibition of the RE-dHP pathway during off-line memory consolidation favors 6-object LTM retention in females by removing inhibitory control over dHP activation, while chemogenetic RE-activation impairs it in males.

Our data represent a first demonstration of a sub-cortical control of dHP recruitment, that might underlie its sex-dependent activation during incidental memory, with potential also for clinical application.
\end{abstract}

One Sentence Summary: The sex-dependent recruitment of a subcortical pathway regulates how many items are spontaneously memorized in mice. 


\section{Introduction}

Memory can be challenged by lengthening the retention interval (its duration) but also by augmenting what has to be retained (its load). Additional neural mechanisms are thought to be necessary as the required duration extends from short (seconds/minutes) to long (hours/days) and to remote (months/years) time scales, based on the evidence that the passage of time promotes the reorganization of neuronal circuits and activates the molecular processes referred to as memory consolidation. The dorsal hippocampus (dHP) has been involved in memory consolidation, the stabilization of a trace from short-term (STM) to long-term memory (LTM) ${ }^{1}$.

However, emerging evidence suggests that also heavier loads, quantified by the number of items that are to be stored in memory, lead to a reorganization of neuronal circuits and to distinct molecular processes. Converging findings in rodents, primates and humans suggest that high memory load drives dHP recruitment in STM item tasks. We and others have previously reported that, in male mice, object STM capacity appears limited to about 6 elements ${ }^{2,3}$, which is very similar to that of humans (albeit measured in different tasks). These findings have been obtained by using the Different/Identical Object Task (DOT/IOT), a modified version of the spontaneous object recognition task, which stimulates incidental encoding based on the spontaneous interest of animals to explore novel objects. In the DOT/IOT, the memory load is manipulated by increasing the number of different objects to remember during the study phase (e.g., 3, 4, 6 and 9 different objects). Lesions of the dHP reduce the STM capacity from 6 to 4 objects ${ }^{2}$.

How the number of elements to remember influences the formation of LTM, namely how duration and load interact, remains a relevant unexplored issue. This is likely because LTM is thought to have almost unlimited capacity, thanks to the possibility of prolonged or multiple trials of learning that facilitate consolidation. However, most of our daily life events are unique experiences, leading to incidental encoding, without necessarily the repetition needed to form stable memories.
Therefore, how the number of elements encountered during incidental encoding affects memory consolidation is an open question. Interestingly, in male mice we have reported that all the 6 objects encoded at short-delay were also remembered at longer delays, through the activation of PKA/CaMKII-mediated GluR1 AMPA receptors phosphorylation at the serine sites S831 and S845 and de novo protein synthesis ${ }^{4}$. These findings in males suggested that all objects encoded in STM are also automatically consolidated into LTM. While studying memory capacity in female mice by subjecting them to the DOT/IOT, however, we discovered that, differently from males, they spontaneously remember at long-delays ( 1 or $24 \mathrm{~h}$ ) only 4 of the 6 objects encoded at short-delay $(1 \mathrm{~min})$, although being more resistant than males to retroactive interference at short-delay (1 min). Though these sex differences do not probably reflect any major qualitative difference in neural mechanism, they offer a precious window to shed light on the dynamic activation of neural circuits, and to study how the duration of the task, far from simply extending the retention of the incidentally acquired items, effectively restricts memory capacity. How does that come about? Sex differences in cognitive functions is an emerging field and their neurobiological bases are still a controversial issue. In the memory domain, sexdifferences have been documented ${ }^{5-7}$, and multiple lines of evidence suggest that in adulthood females tend to engage the dHP less than males, while they tend to prefer to use non-hippocampal dependent navigation strategies when challenged with spatial tasks (reviewed in ${ }^{8}$ ), e.g. while practicing for orienteering competitions 9. Here, we have tested the hypothesis that sexdifferences in the dynamic recruitment of the dHP would influence high load incidental LTM. Additionally, we have used the observed sexdifferences in incidental LTM to provide mechanistic evidence on what drives dHP activation in conditions of high memory load, to help clarify this understudied question in the neurobiology of memory. 


\section{Results}

Female mice do not spontaneously transfer all objects encoded in STM into LTM. While setting up the DOT/IOT in female mice, we found that also females, like males, could remember up to six different objects, but not nine, at short-retention intervals (1 min) (Fig.1 a-d and Extended Data Table 1). However, when female mice were tested 1 or $24 \mathrm{~h}$ after the study phase, their incidental LTM memory span was limited to 4 different objects, unlike that of males, which was 6 (Fig.1e-h). This effect was independent of the phase of the estrous cycle (Extended Data Fig.1a). Thus, while males spontaneously "transfer" all the objects encountered from STM to LTM (1 or $24 \mathrm{~h}$ ), females do the same in condition of low (6-IOT) or intermediate (4-DOT) memory load, but apparently not in the highest memory load conditions (Fig. 2a). Note, though, that they explore the objects more than males during the study phase (Extended Data Table 2). Therefore, in female mice memory stabilization seems not to be automatic but optional, suggesting the possibility of different strategies, or cognitive attitudes, reflected in the high load incidental LTM.

The engagement of LTM mechanisms in condition of high load has been shown to be more resistant, than STM, to retroactive interference ${ }^{10}$. Therefore, we tested "resistance to interference" in both sexes by introducing into the waiting cage a few distracting stimuli (such as other objects and biologically relevant odors) during the $1 \mathrm{~min}$ delay (Fig.1i), a procedure hereon called interference. Exposure to interference disrupted performance in males only in the high load condition (6-DOT) while, surprisingly, it did not affect it at all in females (Fig. 1i'). These data suggest that females, during spontaneous encoding of many objects, can also activate stabilization mechanisms, but in a way that it can make their STM more resistant to retroactive interference and less resistant to the effects of time passing, compared to males. Although these sex differences do not probably reflect structural difference in memory capacity, we have used them as a model to shed light on the dynamic activation of neural circuits that support high load incidental LTM and how it is modulated by sex.

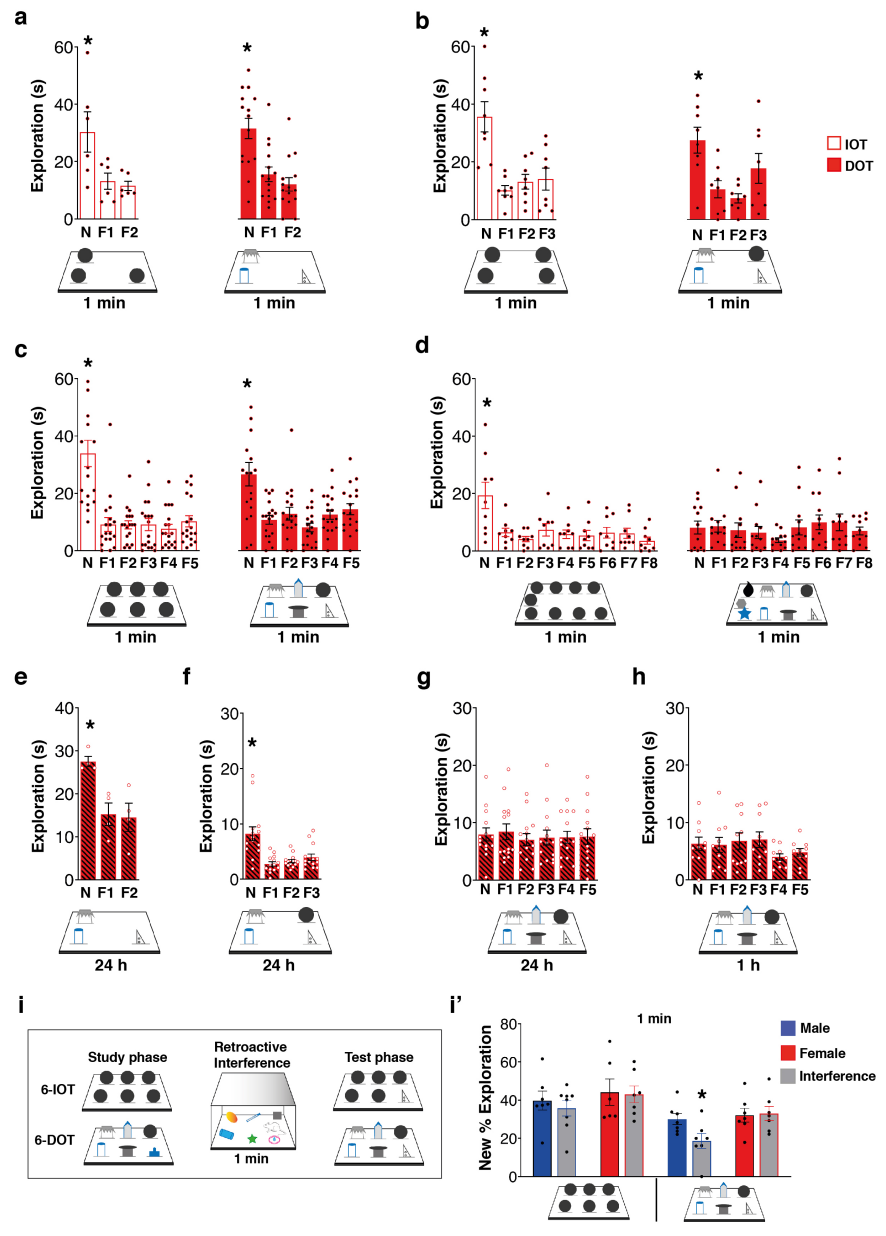

Figure 1. Females show a similar STM capacity to males, but greater resistance to retroactive interference at the expense of reduced memory consolidation. (a-d) Bar charts report exploration of the new object and each of the familiar ones $(\mathrm{F})$ in the Different/Identical Object Task (DOT-IOT). Female mice explored significantly more the new object compared to all the familiar ones at 1 min delay in all the control tasks (IOTs) and in the 3-DOT (a, low load), 4-DOT (b, intermediate load) and 6-DOT (c, high load), but not in the 9-DOT (d, overload). (e-h) In the LTM tasks, females explored significantly more the new object compared to all the familiar ones at $24 \mathrm{~h}$ delay in the 3-DOT (E) and 4-DOT (F), but they showed an impaired performance in the 6-DOT $(\mathrm{G})$, already at $1 \mathrm{~h}$ delay $(\mathrm{H}) .{ }^{*} \mathrm{p}<0.05 \mathrm{New}$ vs all the familiar objects (Dunnett post-hoc). (i-i') Schematic of the task to test the effect of retroactive interference on memory capacity at $1 \mathrm{~min}$ delay (i). No effect of the interference protocol was found on the performance of males and females in the 6-IOT (i'). However, while interference caused a significant decrease of the new $\%$ exploration in males, no effect was revealed in females (i'), which demonstrated to be resistant to retroactive interference even in high memory load condition (6-DOT). ${ }^{*} \mathrm{p}<0.05$ control $v s$ interference within sex. For statistics see Extended Data Table 4.

Female and male mice activate different neuronal circuits in response to a high load memory task. We have tested the hypothesis that males and females use 
different brain circuits when confronted with 6 objects in the DOT task. To explore this hypothesis, we have first conducted a brain mapping study, by measuring the activation of c-Fos protein in different brain regions (as schematized in Fig.2b). We found no differences in the basal level of activation between sexes (Extended Data Table 3). c-Fos expression, as compared to naïve animals, was increased in all the brain regions analyzed except for the infralimbic cortex (IL, Fig. 2c and Extended Data Fig. 2b). The entorhinal (ENT, Fig. 2c and Extended Data Fig. 2d) and perirhinal (PER, Fig. $2 \mathrm{c}$ and Extended Data Fig. 2e) cortices, and the ventral hippocampus (vHP, Fig. 2c and Extended Data Fig. 2g) were similarly activated in both sexes. The 6-DOT induced a significantly higher activation in males than in females for the dHP (Fig. 2c, dashed rectangle on the right), the dentate gyrus (DG, Fig. 2c and Extended Data Fig. 2f), the prelimbic (PL, Fig. 2c and Extended Data Fig. 2a) and anterior cingulate cortices (ACC, Fig. 2c and Extended Data Fig. 2c). On the contrary, the 6DOT induced higher activation in females than in males only in the thalamic nucleus reuniens (RE, Fig. 2c, dashed rectangle on the left).

We have then focused on the dHP and the RE, which showed opposite patterns of activation in males and females. We first verified that the hyperactivation of the $\mathrm{RE}$ and hypoactivation of the $\mathrm{dHP}$ in females compared to males were load-dependent, by comparing their activation in conditions of low (6-IOT), intermediate (3-DOT) and high (6-DOT) memory load (Fig. 2d-e). Based on the existence of reciprocal connections between the dHP and the RE (Extended Data Fig. 3) ${ }^{11,12}$, we performed a Spearman correlational analysis of $\mathrm{RE}$ and dHP activation in the 6-DOT, which resulted positive in both sexes (Fig. 2c'). The RE-dHP correlation plots of males and females clearly showed that, although their activation was correlated in both cases, the average values were different between the two sexes: higher average RE activation levels in females than males corresponded with lower activation values in the dHP (Fig. 2c' '). a

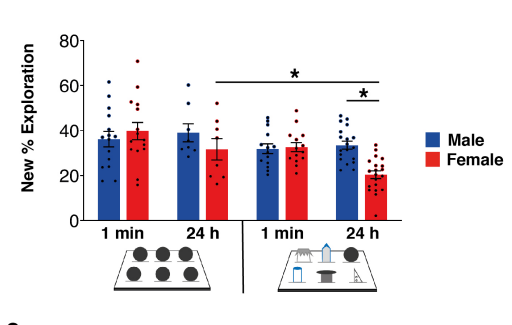

c
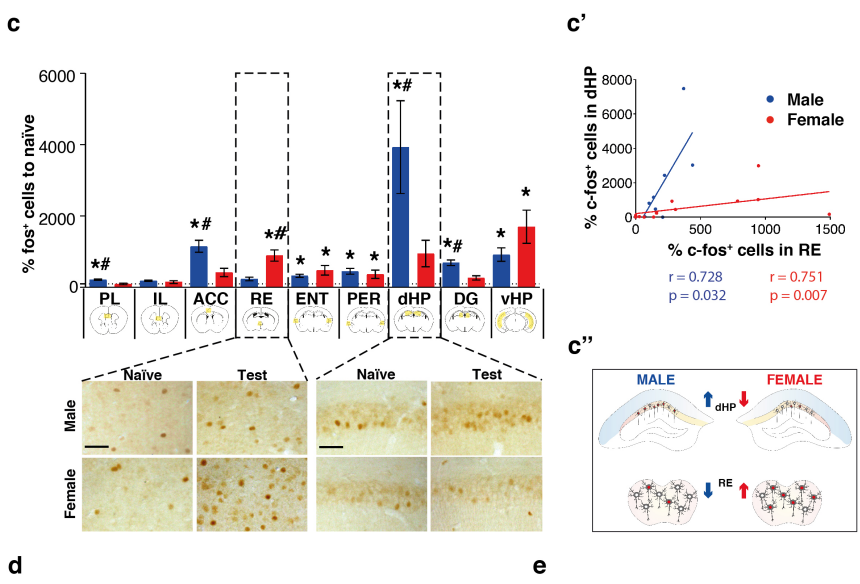

d
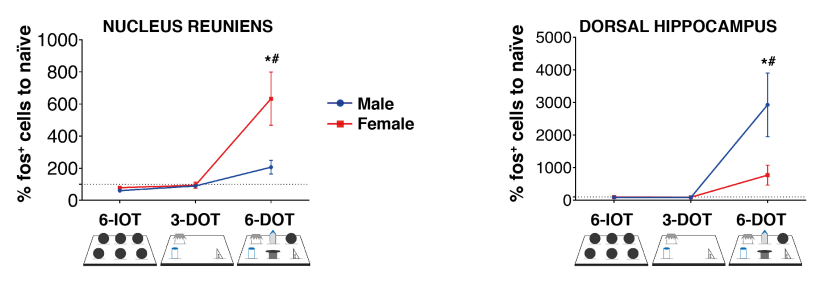

Figure 2. Females show an apparent capacity limit after memory consolidation, associated with RE hyperactivation and dHP hypoactivation compared to males. (a) Bar charts report new object \% exploration in the 6-IOT/DOT for STM (1 min) and LTM $(24 \mathrm{~h})$ of male and female mice. The performance of females in the 6-DOT at $24 \mathrm{~h}$ delay was significantly lower than that in the 6-IOT and that of males in the 6-DOT. (b) Schematic of the c-Fos mapping experiment. (c) Bar charts represent the mean of c-Fos ${ }^{+}$cells normalized on naïve per sex for each brain region analyzed. The RE (dashed square on the left) was the brain region found significantly activated only in females exposed to the 6-DOT. The CA1-CA3 of the dHP (dashed square on the right) was found significantly activated only in male mice exposed to the 6-DOT. The bottom panels show representative photographs of the dHP and the RE in each experimental condition. Scale bar $50 \mu \mathrm{m}$. (c') The Spearman correlation coefficient between RE and dHP c-Fos ${ }^{+}$cells was positive and significant for both sexes. (c') Illustration of the opposite pattern of activation between the dHP and the RE in the two sexes. (d-e) Load-dependent activation of c-Fos in the RE and the dHP for both sexes. The RE was significantly more active in female mice after the 6-DOT compared to lower load conditions (6IOT and 3-DOT) and to males (d). The same happens to the dHP of males (e). Dashed lines represent naïve c-Fos activation. $* \mathrm{p}<0.05$ naïve vs test (within sexes), \# p <0.05 test between sexes 
(Bonferroni correction). For statistics see Extended Data Table 4 and Extended Data Fig. 2.

$\mathrm{PL}=$ prelimbic cortex; IL = infralimbic cortex; $\mathrm{ACC}=$ anterior cingulate cortex; $\mathrm{RE}=$ nucleus reuniens; $\mathrm{ENT}=$ entorhinal cortex; $\mathrm{PER}=$ perirhinal cortex; $\mathrm{dHP}=$ dorsal hippocampus; $\mathrm{DG}=$ dentate gyrus; vHP = ventral hippocampus.

\section{Counteracting RE hyperactivation and dHP} hypoactivation removes the LTM limit in female mice. It is known that the RE can modulate hippocampal excitability ${ }^{12-14}$, thus providing a possible mechanism for engaging/disengaging the dHP in the spontaneous expression of memory observed with the DOT.

To test this hypothesis, we first verified whether the hypoactivation of the dHP and hyperactivation of the $\mathrm{RE}$ have a functional role in the DOT memory observed in females, by using Designer Receptors Exclusively Activated by Designer Drugs (DREADDs) (Fig. 3a). The chemogenetic stimulation of the dHP and inhibition of the RE both removed the apparent capacity limit in females (Fig. 3b-b" and Fig. 3c-c"'; Extended Data Fig. 4a-b) and, remarkably, increased the phosphorylation of the serine site S845 (p-S845) of the GluR1 subunit of the AMPA receptor in the dHP (Fig. $\left.3 b^{\prime}-c^{\prime}\right)$, which is a mechanism required for processing high memory load in males ${ }^{4}$. Importantly, this effect was the first evidence we obtained that the inhibition of the RE could rescue the dHP activation in females (Fig. $3 b$ '). We excluded that these effects were due to the injection of the clozapine-N-oxide (CNO) per se, as the same dose of CNO injected in naïve female mice did not affect either memory or p-S845 (Extended Data Fig. 4d-d'). Moreover, we showed that the dHP chemogenetic activation effect was time-dependent, because injection of CNO immediately post-training was ineffective (Extended Data Fig. 4e-e'), and the pharmacokinetic of CNO indicates that in the previous experiment it would have acted approximately 35 minutes after the beginning of early memory consolidation ${ }^{15}$. This finding shows that in order to remove the apparent capacity limit, hippocampal activation must be induced in the early phase of memory consolidation.
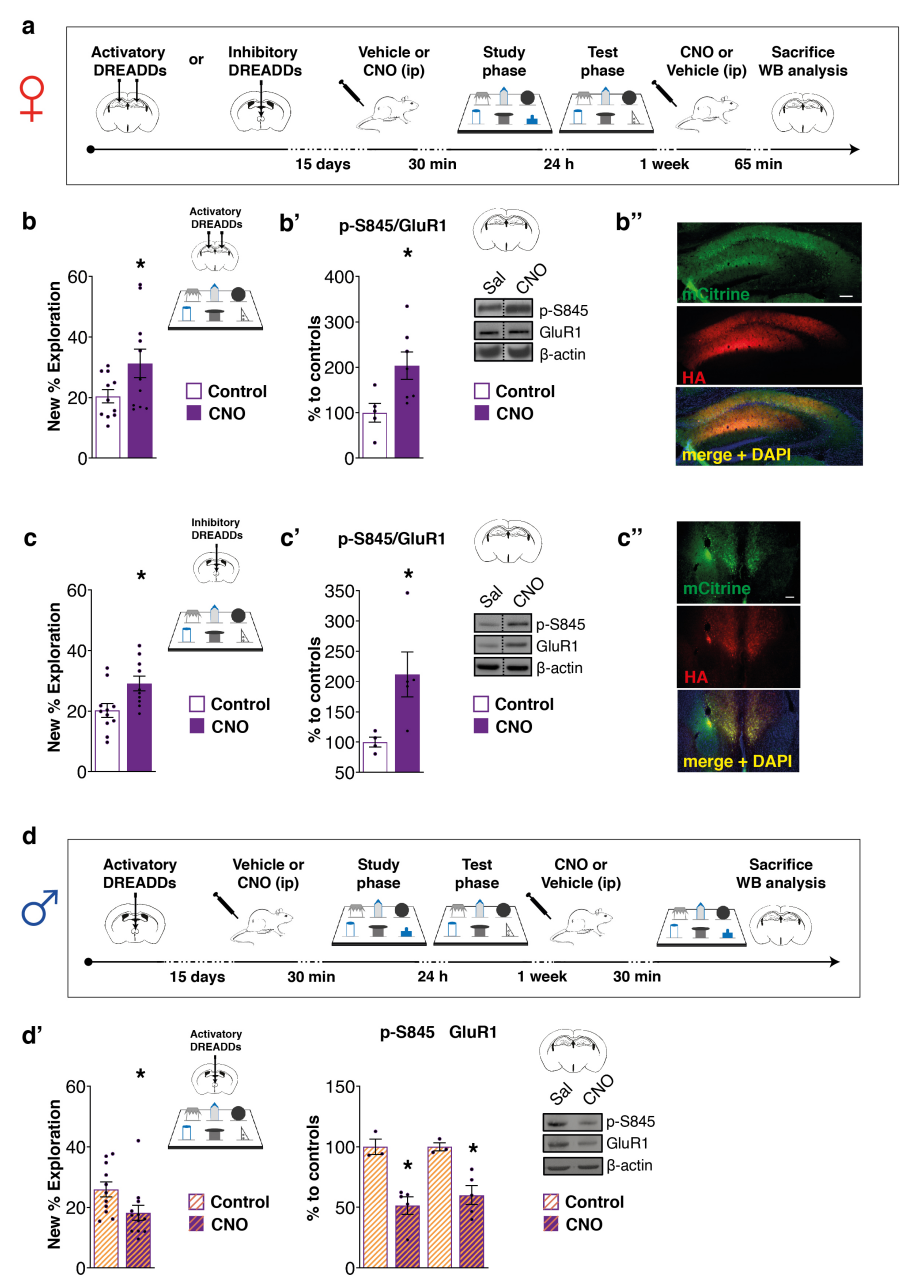

Figure 3. Activation of the $\mathrm{dHP}$ and inhibition of the RE rescues the expression of high load incidental LTM. (a) Schematic of the experimental design showing the timing of injection of an activatory DREADDs in the dHP or an inhibitory one in the RE of female mice and the experimental course. (b) AAV-CaMKIIa-HA-rM3D(Gs)IRES-mCitrine was injected in the dHP of females. Bar chart represents increased new object \% exploration for $\mathrm{CNO}$ treated females compared to controls. (b') Bar charts represent western blot analysis of p-S845/GluR1 levels in the dHP of vehicle and CNO treated mice, which is increased by dHP chemogenetic activation. No change was detected in the expression of GluR1. On the right are shown representative bands for each condition. (b') Representative images of AAV expression in the dHP; scale bar 100 $\mu \mathrm{m}$. (c) AAV-CaMKIIa-HA-rM4D(Gi)-IRES-mCitrine was injected in the RE of female mice. Bar charts represent increased new object \% exploration in $\mathrm{CNO}$ treated females compared to controls. (c') Bar charts represent western blot analysis of pS845/GluR1 levels in the hippocampus of vehicle and CNO treated mice, which shows a significant increase after RE inhibition. Concomitant we found that RE inhibition also increased the GluR1 levels. On the right are shown representative bands for each condition. (c') Representative images of AAV expression in the dHP; scale bar $100 \mu \mathrm{m}$. (d) Schematic of the experimental design 
showing the timing of injection of an activatory DREADDs in the RE of male mice and the experimental course. (d') AAV-CaMKIIaHA-rM3D(Gs)-IRES-mCitrine was injected in the RE of males. Bar charts on the left represent new object \% exploration for vehicle and CNO treated mice showing that RE activation induced a memory consolidation capacity limit in males. Bar charts on the right represent western blot analysis of p-S845 and GluR1 levels in the $\mathrm{dHP}$ of vehicle and CNO treated mice, which were both decreased by the chemogenetic activation of the RE, while no change was detected on their ratio. Representative bands for each condition are shown on the right. $* \mathrm{p}<0.05$ between groups. For statistics see Extended Data Table 4.

\section{Optogenetic inhibition of the RE-dHP pathway also promotes dHP activation, removing the high load} incidental LTM limit in female mice. We next asked whether the limit on the spontaneous memory expression associated with the hyperactivation of the RE is sex-specific. To address this issue, we "feminized" the RE of a group of male mice by chemogenetically stimulating it (Fig.3d). This approach led to the same apparent limit as in females and decreased the level of p-S845 and GluR1 after exposure to the 6-DOT compared to controls (Fig. 3d').

The data obtained so far suggested that RE hyperactivation negatively modulates the $\mathrm{dHP}$, and that in females the high load of the 6-DOT naturally triggers this condition resulting in dHP hypoactivation. To confirm this hypothesis, we used an optogenetics approach aimed at selectively inhibiting the RE-dHP direct pathway. We first injected an adeno-associated viral vector expressing Halorhodopsin (eNpHR3.0) into the $\mathrm{RE}$ and showed that in females, continuous photoinhibition of the RE post-learning was sufficient to rescue the expression of spontaneous memory in the 6-DOT (Extended Data Fig. 5a-a'). Then, we injected eNpHR3.0 in the RE and placed the fiber optic cannulae into the dHP in order to inhibit specifically the RE-dHP pathway (Fig. 4a).

Using three parallel approaches, we showed that in females, photoinhibition of the RE-dHP pathway: 1) increased the frequency and amplitude of the excitatory post synaptic currents (EPSPs) in the dHP, as measured by ex-vivo patch clamp recordings in hippocampal slices (Fig. 4b-b'); 2) increased the spontaneous extracellular multiunit activity (MUA) in the pyramidal layer of the CA1 region recorded in vivo in anaesthetized mice (Fig. 4b-b'); 3) removed the apparent memory limit in the 6-DOT (Fig. 4c-c') and increased 6-DOT-induced c-Fos in the dHP (Fig. 4dd'). The same approaches had no effects on dHP activity in naïve (not injected) animals (Extended Data Fig. 5b) and in males (Extended Data Fig. 5c-d'), indicating that RE-dHP pathway is likely silent in male mice in basal conditions (Extended Data Fig. 5c-c'').

We also measured in the stratum oriens (so) of the dHP the number of total c-Fos ${ }^{+}$cells and those that colocalized with GAD (Fig. 4e-f), a marker of inhibitory neurons, to evaluate any potential change in the inhibitory tone of the dHP in females. The total amount of c-Fos ${ }^{+}$cells in the so was increased, similarly to what we observed in $p y$; in parallel, the percentage of colocalizing $\mathrm{GAD}^{+} / \mathrm{c}-\mathrm{Fos}^{+}$cells in so decreased after RE-dHP photoinhibition (Fig. 4f-f'). These data suggested that the inhibition of the RE-dHP leads to increased hippocampal activity.

RE-dHP photoinhibition also increased the expression of the eukaryotic translation initiation factor EIF2 $\alpha$, an early regulator of protein synthesis initiation, and decreased the ratio of its phosphorylation (Fig.4g-g'), $1 \mathrm{~h}$ after the end of the 6-DOT, which can be considered as an index of increased learning-associated protein synthesis ${ }^{16}$.

These results indicate that in females, inhibition of REterminals increases glutamatergic transmission in the dHP, possibly by a disinhibitory mechanism, thereby allowing the activation of the LTM molecular processes that males use to allow the expression of spontaneous LTM in conditions of high memory load. 

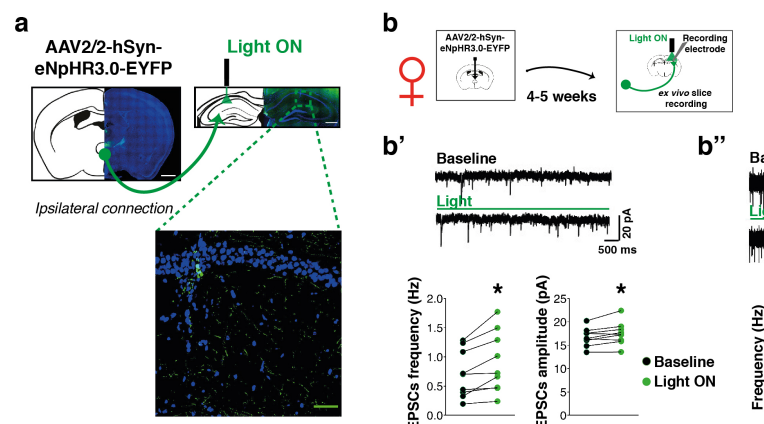

b'

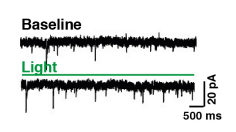

b"

Baseline

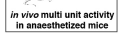
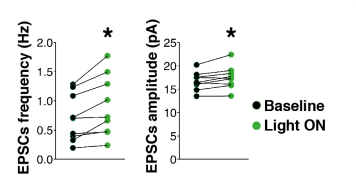

c'
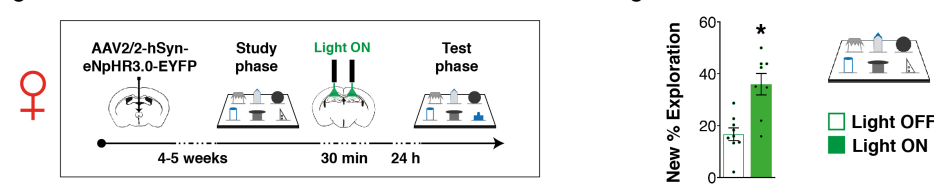

d Light OFF

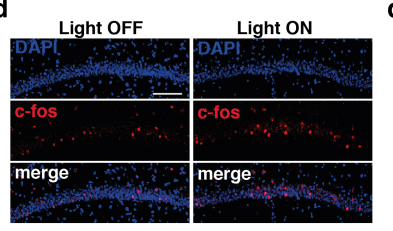

d'

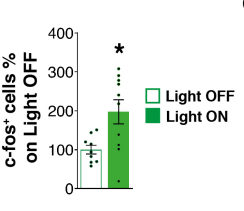

e c-fos $/ \mathrm{GAD}^{*}$ neuron in so

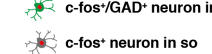

c-fos neuron in py

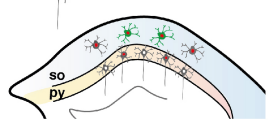

f

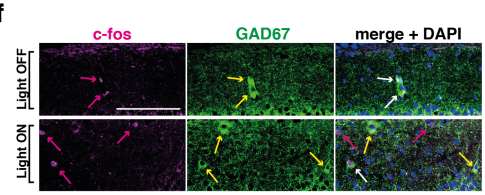

f'

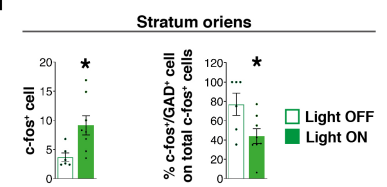

g
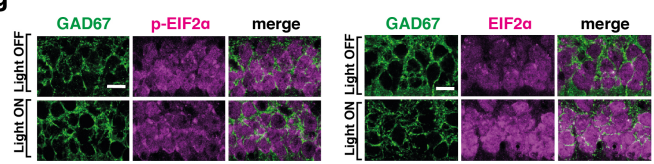

g'

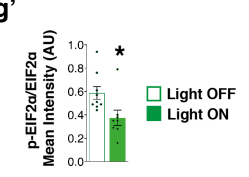

Figure 4. RE-dHP photoinhibition increases hippocampal activity and rescues high load incidental LTM in females. (a) Representative images of the AAV injection in the RE and its diffusion in the fiber terminals at the level of the dHP (scale bar 100 $\mu \mathrm{m}$ and $50 \mu \mathrm{m}$ in the squared magnification). (b) Schematic of the experimental plans: AAV-hSyn-eNpHR3.0-EYFP was injected in the RE and after about 4-5 weeks mice underwent ex vivo recordings in hippocampal slices (b') or in vivo hippocampal multiunit activity recordings under anesthesia (b'). (b') On the top, representative traces from CA1 hippocampal neurons showing EPSCs in baseline and under photoinhibition (green bar). On the bottom, scatter plot showing EPSPs increased frequency and amplitude for females. (b') On the top, representative traces of multiunit activity recorded from hippocampal CA1 pyramidal layer before and during photoinhibition (green bar). On the bottom, bar charts represent increased frequency activity in females between baseline (control) and under photoinhibition (light $\mathrm{ON}$ ). $* \mathrm{p}<0.05$ between groups. (c) Schematic of the experimental design showing the timing of REdHP fibers post-training (30 min) continuous inhibition. (c') Bar charts represent the new object \% exploration in light off and light on females in the test conducted $24 \mathrm{~h}$ after post-training RE-dHP photoinhibition, which shows a rescued memory consolidation capacity. (d-d') RE-dHP photoinhibition increased c-Fos ${ }^{+}$cells in the CA1 pyramidal layer of females; scale bars: $100 \mu \mathrm{m}$. (e)
Schematic of the dHP pyramidal layer (py) and stratum oriens (so) in which the c-Fos ${ }^{+}$cells and the co-localizing signal with GAD67 $\left(\mathrm{GAD}^{+}\right.$cells) was analysed. (f-f') RE-dHP photoinhibition also increased c-Fos ${ }^{+}$cells in the so of the CA1, but the percentage of $\mathrm{GAD}^{+} / \mathrm{c}-\mathrm{Fos}^{+}$cells was lowered by RE-dHP photoinhibition; scale bar $100 \mu \mathrm{m}$; magenta arrows indicate c-Fos ${ }^{+}$cells, yellow arrows $\mathrm{GAD}^{+}$ones, while white arrows indicate c-Fos ${ }^{+} / \mathrm{GAD}^{+}$cells. (g-g') Quantitative analyses of immunofluorescence intensity in the CA1 excitatory neurons after RE-dHP photoinhibition showed decreased p-EIF2 $\alpha /$ EIF $2 \alpha$ ratio in females; scale bar $10 \mu \mathrm{m} . * \mathrm{p}<0.05$ between groups. For statistics see Extended Data Table 4.

\section{Discussion}

Our study shows that both female and male mice express spontaneous memory for up to about 6 different objects at short time delays (1 min). However, keeping this memory load with longer retention intervals (1 to $24 \mathrm{~h}$ ) unveiled an incidental LTM limit in female, but not male mice. Of note, we are not investigating the capacity of LTM, but how the memory load influences memory consolidation during spontaneous encoding. Our spontaneous task reveals sex differences in the engagement dynamics of those circuits, in particular in the LTM manifestation of clear STM traces. Is it that not all of the information registered in STM is stabilized into LTM? Studies performed in males may be taken to indicate that high memory load encourages cooperation between STM and LTM mechanisms in the brain ${ }^{4}$; indeed, high memory load favors dHP recruitment in STM tasks. This raises the interesting question of what drives hippocampal recruitment in high memory load tasks? Here, we report a subcortical-to-cortical mechanism that controls the LTM, preventing it in females under high memory load, through RE hyperactivation, a mechanism which can be exogenously imposed also onto males. Indeed, while males normally recruit the dHP, which favors the expression of LTM traces, females hyperactivate the $\mathrm{RE}$, which by lowering the dHP activation, limits memory consolidation. The fact that chemogenetic activation of the dHP is sufficient to remove the apparent LTM limit in females strengthens the idea that high memory load somehow promotes cooperation between STM and LTM mechanisms through the engagement of the $\mathrm{dHP}^{4}$. It is possible that the same $\mathrm{RE}$ control mechanism operates naturally in males as well, but in conditions of even higher memory load. Indeed, in a previous study in males we reported that 
increasing the number of objects from 1 to 6 was associated to progressive increase in p-GluR1; however, with 9 objects (an overload condition) pGuR1 drops to the basal level, despite animals exploring the objects during the study phase, suggesting a lack of recruitment of the dHP.

Previous evidence in the literature shows that biological relevant stimuli (such as fear), complex tasks and fear memory extinction ${ }^{17-22}$ all lead to RE activation, which regulates learning and memory consolidation when the interaction between the prefrontal cortex and the dHP is required ${ }^{23-25}$. Our findings add to this body of data, showing that the RE activation is also determined by information load and that its level of activation is tuned differently in the two sexes. It highlights the importance of memory load as a new, and as yet unexplored, factor governing memory consolidation for unique experiences; indeed, we cannot exclude that multiple training session might eliminate or revert the identified sex-differences in spontaneous memory. The fact that the degree of RE activation, which in our study we define as "hyper" vs "hypo" active, is a determinant of the dHP-dependent response is not completely new. Indeed, our evidence is in line with previous data showing that the pattern of stimulation of the RE is a relevant factor for determining the net effect on dHPdependent memory also in males: in vivo tonic and phasic optogenetic stimulation of the RE during the acquisition of contextual fear conditioning reduced or augmented fear memory generalization, respectively ${ }^{26}$.

From the neurobiological perspective, one possible explanation of the sexually dimorphic action of the RE on dHP activation, in response to the 6-DOT, is that RE hyperactivation in females stimulates dHP GABAergic interneurons more than in males. This might be a consequence of the different pattern of RE stimulation of the dHP, which cannot be distinguished by c-Fos activation alone. Another possibility is that males may have a more resistant dHP activity, as suggested by reports of stronger long-term potentiation and hippocampal theta power activity in males compared to females ${ }^{27,28}$. A higher dHP resistance might in turn limit RE activation through feedback modulation ${ }^{14}$. These hypotheses are also compatible with the lack of effect of RE-dHP optogenetic inhibition on the frequency of EPSPs in the dHP of males, as well as on their memory consolidation, in line with previous reports $22,24,25,29,30$.

Sex-dependent differences in dHP recruitment have already been identified using different behavioral tasks ${ }^{8}$, but the mechanism underlying the reduced tendency of females to activate the dHP was unknown. Most of the evidence indicates a top-down control of memory functions ${ }^{31,32}$. Our data represent a first demonstration of sub-cortical control of dHP recruitment, which might underlie the sex-regulated activation of the dHP, with high potential also for clinical application.

The negative thalamic regulatory control of dHP activation in high memory load conditions and the consequent limit in the expression of spontaneous memory should not be seen as a structural limit in females, as it may allow them to focus better on ongoing cognitive tasks - possibly, it is what makes them more resistant to retroactive interference. These hypothesis is in line with previous studies showing that thalamic activation sustains attentional control ${ }^{33}$.

The role of thalamic control on cortical activation in memory and cognition is of great current interest. Our data are in line with a recent finding in humans indicating an anti-correlated activation of the mediodorsal thalamus and the hippocampus specifically in females, but not males ${ }^{34}$. We report sex differences in such control mechanisms and demonstrate the existence of these differences in the functional recruitment of the dHP under high memory load, leading in females to limit the expression of spontaneous memory.

The novel findings that we report suggest a major interaction between subcortical and cortical regions in determining memory processes and show that studying sex differences is not only necessary for sex-addressed therapeutic strategies, but also is a key for elucidating new neurobiological mechanisms of cognitive functions. 
Acknowledgments: Authors would like to thank De Leonibus' lab members, Dr. Elizabeth Illingworth, Dr. Cornelius Gross, Dr. Vivien Chevaleyre and Prof. Enrico Cherubini for critical revising the manuscript and Dr. Phoebe Ashley-Norman for English editing. Authors would also like to thank Miriam Cerullo who helped with RE-dHP activity correlation and loaddependent activation experiments and Martina Colucci who helped with the immunohistochemistry counting. Funding: This study was supported by the Grant "Neurobiology of sex differences influencing memory capacity in ageing and AD" (SAGA-17-418745) from the Alzheimer's Association to EDL.

Author contributions: G.T. performed behavioral and immunofluorescence experiments and mice surgeries, conducted all data analysis and statistics, wrote the first draft of the manuscript, reviewed and edited the manuscript, conceptualized optogenetics experiments. V.L. performed behavioral, western blot, immunohistochemistry and immunofluorescence experiments, performed virus localization experiments, conducted data analysis and statistics, produced AAVs for optogenetics, contributed to the review and editing of the manuscript. D.C. performed behavioral and immunofluorescence experiments, contributed to data analysis and statistics, contributed to the review and editing of the manuscript. F.E. performed behavioral and immunohistochemistry experiments. A.C. performed and supervised part of the immunofluorescence experiments. M.Gi. contributed to set up optogenetics. M.D.R. performed western blot experiments and produced AAVs for DREADDs, contributed to the review and editing of the manuscript. A.T. contributed to the interpretation of the results and the editing of the manuscript. M.G. conceptualized and performed electrophysiology experiments, contributed to the review and editing of the manuscript. EDL conceived and supervised the study and the experiments, acquired funding, wrote the manuscript. All authors contributed to the discussion and approved the manuscript and supporting information.
Competing interests: Authors declare no competing interests.

\section{References}

1. Frankland, P. W. \& Bontempi, B. The organization of recent and remote memories. Nat. Rev. Neurosci. 6, 119-30 (2005).

2. Sannino, S. et al. Role of the dorsal hippocampus in object memory load. Learn. Mem. 19, 211-218 (2012).

3. Sugita, M., Yamada, K., Iguchi, N. \& Ichitani, Y. Hippocampal NMDA receptors are involved in rats' spontaneous object recognition only under high memory load condition. Brain Res. 1624, 370-379 (2015).

4. Olivito, L. et al. Phosphorylation of the AMPA receptor GluA1 subunit regulates memory load capacity. Brain Struct. Funct. 221, 591-603 (2016).

5. Spets, D. S., Jeye, B. M. \& Slotnick, S. D. Different patterns of cortical activity in females and males during spatial long-term memory. Neuroimage 199, 626-634 (2019).

6. Spets, D. S. \& Slotnick, S. D. Are there sex differences in brain activity during long-term memory? A systematic review and fMRI activation likelihood estimation meta-analysis. Cogn. Neurosci. 00, 1-11 (2020).

7. Cimadevilla, J. M., Conejo, N. M., Miranda, R. \& Arias, J. L. Sex differences in the Morris water maze in young rats: Temporal dimensions. Psicothema 16, 611-614 (2004).

8. Torromino, G., Maggi, A. \& Leonibus, E. De. Estrogen-dependent hippocampal wiring as a risk factor for age-related dementia in women. Prog. Neurobiol. doi:10.1016/j.pneurobio.2020.101895

9. Juhas, I., Baĉanac, L. \& Kozoderović, J. The Most Common Errors in Orienteering and Their Relation To Gender, Age and Competition Experience. Facta Univ. Ser. Phys. Educ. Sport 14, 211-226 (2016).

10. Shrager, Y., Levy, D. A., Hopkins, R. O. \& Squire, L. R. Working Memory and the 
Organization of Brain Systems. 28, 4818-4822 (2008).

11. Dolleman-Van Der Weel, M. J. \& Witter, M. P. Projections From the Nucleus Reuniens Thalami to the Entorhinal Cortex , Hippocampal Field CA1, and the Subiculum in the Rat Arise From Different Populations of Neurons. $J$. Comp. Neurol. 364537450, (1996).

12. Dolleman-van DerWeel, M. J. \& Witter, M. P. Nucleus reuniens thalami innervates $g$ aminobutyric acid positive cells in hippocampal field CA1 of the rat. Neurosci. Lett. 278, 145148 (2000).

13. Bertram, E. H. \& Zhang, D. X. Thalamic excitation of hippocampal CA1 neurons: A comparison with the effects of CA3 stimulation. Neuroscience 92, 15-26 (1999).

14. Dolleman-van DerWeel, M. J. et al. The nucleus reuniens of the thalamus sits at the nexus of a hippocampus and medial prefrontal cortex circuit enabling memory and behavior. Learn. Mem. 26, 191-205 (2019).

15. Roth, B. L. DREADDs for Neuroscientists. Neuron 89, 683-694 (2016).

16. Sharma, V. et al. eIF2 $\alpha$ controls memory consolidation via excitatory and somatostatin neurons. Nature (2020). doi:10.1038/s41586020-2805-8

17. Ramanathan, K. R., Ressler, R. L., Jin, J. \& Maren, S. Nucleus reuniens is required for encoding and retrieving precise, hippocampaldependent contextual fear memories in rats. $J$. Neurosci. 38, 9925-9933 (2018).

18. Silva, B. A., Burns, A. M. \& Gräff, J. A cFos activation map of remote fear memory attenuation. Psychopharmacology (Berl). 236, 369-381 (2019).

19. Silva, B. A. et al. A thalamo-amygdalar circuit underlying the extinction of remote fear memories. Nat. Neurosci. (2021). doi:10.1038/s41593-021-00856-y

20. Lin, Y. J., Chiou, R. J. \& Chang, C. H. The reuniens and rhomboid nuclei are required for acquisition of pavlovian trace fear conditioning in rats. eNeuro 7, 1-15 (2020).

21. Loureiro, M. et al. The ventral midline thalamus (Reuniens and rhomboid nuclei) contributes to the persistence of spatial memory in rats. $J$. Neurosci. 32, 9947-9959 (2012).

22. Barker, G. R. I. \& Warburton, E. C. A critical role for the nucleus reuniens in long-term, but not short-term associative recognition memory formation. J. Neurosci. 38, 3208-3217 (2018).

23. Ito, H. T., Zhang, S., Witter, M. P., Moser, E. I. \& Moser, M. A prefrontal-thalamohippocampal circuit for goal-directed spatial navigation. Nature 522, (2015).

24. Cholvin, T., Hok, X. V., Giorgi, L., Chaillan, F. A. \& Poucet, X. B. Ventral Midline Thalamus Is Necessary for Hippocampal Place Field Stability and Cell Firing Modulation. $J$. Neurosci. 38, 158-172 (2018).

25. Hauer, B. E., Pagliardini, S. \& Dickson, C. T. The reuniens nucleus of the thalamus has an essential role in coordinating slow-wave activity between neocortex and hippocampus. eNeuro 6, (2019).

26. Xu, W. \& Südhof, T. C. A Neural Circuit for Memory Specificity and Generalization. Science. 339, 1290-1296 (2013).

27. Maren, S., De Oca, B. \& Fanselow, M. S. Sex differences in hippocampal long-term potentiation (LTP) and Pavlovian fear conditioning in rats: positive correlation between LTP and contextual learning. Brain Res. 661, 25-34 (1994).

28. Juárez, J. \& Corsi-Cabrera, M. Sex differences in interhemispheric correlation and spectral power of EEG activity. Brain Res. Bull. 38, 149-151 (1995).

29. Ito, H. T., Moser, E. I. \& Moser, M. Supramammillary Nucleus Modulates SpikeTime Coordination in the Prefrontal-ThalamoHippocampal Circuit during Navigation Article Supramammillary Nucleus Modulates SpikeTime Coordination in the Prefrontal-ThalamoHippocampal Circuit during Navigation. Neuron 99, 576-587.e5 (2018).

30. Dolleman-Van Der Weel, M. J., Morris, R. G. M. \& Witter, M. P. Neurotoxic lesions of the thalamic reuniens or mediodorsal nucleus in rats affect non-mnemonic aspects of watermaze learning. Brain Struct. Funct. 213, 329-342 (2009). 
31. Torromino, G. et al. Offline ventral subiculumventral striatum serial communication is required for spatial memory consolidation. Nat. Commun. 10, 1-9 (2019).

32. Vetere, G. et al. An inhibitory hippocampalthalamic pathway modulates remote memory retrieval. Nat. Neurosci. 24, 685-693 (2021).

33. Schmitt, L. I. et al. Thalamic amplification of cortical connectivity sustains attentional control. Nat. Publ. Gr. 545, (2017).

34. Spets, D. S. \& Slotnick, S. D. Thalamic Functional Connectivity during Spatial LongTerm Memory and the Role of Sex. Brain Sci. 10, 1-15 (2020).

\section{Methods}

\section{Subjects}

In this study we used outbred CD1 adult (3 months old) male and female mice (Charles River, Italy) housed in groups of 3-5 subjects.

Housing conditions were maintained at $22 \pm 1{ }^{\circ} \mathrm{C}$, with relative humidity of $55 \pm 5 \%$, and with a $12 \mathrm{~h}$ light/12 h dark cycle (light: 7:00 am - 7:00 pm). Mice were always tested during the light phase.

Before behavioral procedures, the estrous cycle of females was checked through a visual method ${ }^{35}$, chosen because of its low invasiveness in order to avoid stress to the animals. Females were tested in all the phases of estrous cycle except the estrous, unless specified.

All procedures were performed in strict accordance with the European Communities Council directives and Italian laws on animal care. Experiments were performed under blind to treatment and sex conditions, unless specified.

\section{Habituation procedure}

Before each behavioral procedure, mice were first subjected to a habituation procedure. Briefly, the procedure began with manipulation of the mice for 2-5 min. Then, mice were allowed to acclimatize to a personal waiting cage for $10 \mathrm{~min}$. The entire procedure was repeated once a day for at least one week before the beginning of the behavioral procedure.

\section{Different/Identical Objects Task}

The Different/Identical Objects Task (DOT/IOT) was performed as described by Sannino et al., 2012. Briefly, animals were isolated for $15 \mathrm{~min}$ in a personal waiting cage and then subjected to a familiarization trial $(\mathrm{T} 1 ; 10$ $\mathrm{min})$ in an empty open field ( $35 \times 47 \times 60 \mathrm{~cm})$. After 1 min spent in their personal waiting cage, mice were subjected to the study phase (T2; $10 \mathrm{~min})$, during which they were allowed to explore the objects until they accumulated $35 \mathrm{sec}$ for the IOT or $35 \mathrm{sec}$ per different object for the DOT. Exploration was defined as the time in which the nose of the mouse was in contact with or less than $2 \mathrm{~cm}$ from the object ${ }^{36}$.

After a delay of $1 \mathrm{~min}$, for the short-term memory (STM) task, or 1 or $24 \mathrm{~h}$ for the long-term memory (LTM) task, animals underwent testing, in which they were exposed to identical copies of the familiar objects and to a completely new object (T3; $5 \mathrm{~min}$ ). Two different new objects were alternatively used between animals, and the position of the new object was changed across animals in a random manner to avoid any bias linked to the object used or to its position.

For c-Fos male/female comparison experiments, total exploration of females was matched to that of males.

For DREADDs and optogenetics experiments the same animals were tested two times (15 days apart) and treatments (saline or CNO, light off or light on) were counterbalanced for each test.

The behavior of the animals was recorded by a videotracking system (Anymaze, Stoelting, USA) and analyzed by a trained observer. We performed off-line behavioral scoring to keep the experimenters blind to the treatments and sex of the animals; when this was not possible, we performed double blind random re-scoring performed by another experiments fully blind on the experimenter and obtained comparable scoring results. Results are expressed as discrimination index, that is time spent exploring the new object as a percentage of the total exploration time ${ }^{36}$.

\section{Retroactive interference procedure}

The 6-IOT/DOT was performed as described before. Mice from the interference group were put in the interference cage during the delay between $\mathrm{T} 2$ and $\mathrm{T} 3$. The interference cage consisted of a standard plastic cage $(42.5 \times 26.6 \times 18.5 \mathrm{~cm})$ filled with small size objects different from those explored in the T2, of which some were embedded with biologically relevant odors (food, sawdust from pups cage).

\section{c-Fos brain mapping by immunohistochemistry}

Male and female mice were exposed to the habituation and the study phase of the 6-DOT (high memory load) and their total object exploration was matched (females to males). Handled naïve sex-matched mice were used 
as a control group and for normalization of data. All mice were handled as previously described.

After $1 \mathrm{~h}$, in order to ensure c-Fos protein expression ${ }^{37-}$ 39 , mice were deeply anaesthetized and transcardially perfused with $1 \mathrm{x}$ PBS followed by $4 \%$ paraformaldehyde (PFA; Sigma Aldrich). Brains were collected and post-fixed for $24 \mathrm{~h}$ in PFA and passed in a $30 \%$ sucrose solution. $30 \mu \mathrm{m}$ coronal slices were obtained with the use of a vibratome (Leica VT1000 S) and stored in PBS and sodium azide $(0.02 \%)$ at $4^{\circ} \mathrm{C}$ until histological processing.

4 to 7 free-floating sections per animal were chosen considering the antero-posterior extent for each brain region analysed.

After rinsing in $1 \mathrm{x}$ PBS, brain sections were preincubated with $0.5 \% \mathrm{H}_{2} \mathrm{O}_{2}$ in $100 \%$ ethanol for 20 minutes to block intrinsic peroxidase activity. Sections were then washed three times with 1x PBS before being pre-incubated for $1 \mathrm{~h}$ in a blocking solution made of PBS $0.3 \%$ Triton X-100, 1\% BSA and 5\% normal goat serum (NGS). Blocking solution was then removed and slices were incubated for three days at $4{ }^{\circ} \mathrm{C}$ with a solution containing $2 \%$ NGS, PBS $0.3 \%$ Triton X-100 and 1:400 rabbit anti-c-Fos antibody (sc-52; Santa Cruz Biotechnology). Sections were washed three times in PBS Triton $0.3 \%$ (PBS-T) and then incubated for $2 \mathrm{~h}$ with anti-rabbit IgG peroxidase-labeled diluted 1:300 in PBS-T containing 1\% NGS. Brain sections were exposed to avidin-biotin complex (Avidin/Biotin Blocking Kit; SP-2001, Vector Laboratories, Burlingame, CA). The staining was visualized by color reaction with 3,3' diaminobenzidinetetrahydrochloride (DAB Peroxidase HRP Substrate Kit, 3,3'diaminobenzidine; SK-4100; Vector Laboratories, Burlingame, CA) under microscopic control until optimal staining was achieved (approximately 2-5 $\mathrm{min})$. The color reaction was terminated by rinsing sections in Milli-Q water overnight. Finally, sections were mounted on microscope glass slides and coverslipped with Mowiol (4-88; Sigma Aldrich). Control sections, which had not been exposed to primary antibody, were processed in parallel.

Images (5x magnification) were taken using the microscope Leica DM6000B with Leica digital camera DFC 480 RGB and Leica application Suite X (LAS X) software.

The number of c-Fos positive cells were manually counted with ImageJ software (National Institute of Health, Bethesda, MD) in all the brain structures for each slice per animal. Number of cells per slice were averaged per group of antero-posterior coordinates and then averaged for each animal. Considering the antero- posterior extent, the resulting values per animal were normalized on naïve average and expressed as percentage of naïve.

\section{Preparation of AAVs}

Plasmids for DREADDs and optogenetic experiments were purchased from Addgene.

For DREADDs experiments we used pAAV-CaMKIIaHA-rM3D(Gs)-IRES-mCitrine (\#50468) and pAAVCaMKIIa-HA-hM4D(Gi)-IRES-mCitrine (\#50467).

For optogenetic experiments we used pAAV-hSyneNpHR 3.0-EYFP (\#26972).

Plasmids were amplified and purified using EndoFree Plasmid Mega Kit (Qiagen), following manufacturer's instruction and AAVs were assembled by the TIGEM AAV Vector Core.

AAV serotypes produced resulted in the following titer $(\mathrm{GC} / \mathrm{ml}): 1.4 \times 10^{12}$ for AAV 2/5-CaMKIIa-HArM3D(Gs)-IRES-mCitrine, $2.1 \times 10^{12}$ for AAV 2/5CaMKIIa-HA-hM4D(Gi)-IRES-mCitrine and $8.4 \mathrm{x}$ $10^{12}$ for AAV 2/2-h-SYN-eNpHR3.0-EYFP.

\section{Stereotaxic surgery}

For virus injection, mice were anaesthetized with a mixture of tiletamine/zolazepam (Zoletil; $80 \mathrm{mg} / \mathrm{Kg}$ ) and xilazine $(10 \mathrm{mg} / \mathrm{Kg})$ and placed on a stereotaxic apparatus (Stoelting, USA). The skull was exposed and small craniotomies were made at the following coordinates: antero-posterior (AP) $-0.82 \mathrm{~mm}$ and mediolateral $(\mathrm{ML}) \pm 0.2 \mathrm{~mm}$ from bregma, and dorsoventral (DV) $-4.3 \mathrm{~mm}$ from the dura for the nucleus reuniens (RE); AP $-1.80 \mathrm{~mm}, \mathrm{ML} \pm 1.5 \mathrm{~mm}$ from bregma and DV $-1.6 \mathrm{~mm}$ from the dura for the hippocampus (HP). Injection volume was $0.4 \mu \mathrm{L}$ for the $\mathrm{RE}$ and $0.5 \mu \mathrm{L}$ per side for the HP.

For fiber optic implantation, during the same surgery one or two $200 \mu \mathrm{m}$ core $(0.39 \mathrm{NA})$ optic fibers were implanted at the coordinates AP $-0.82 \mathrm{~mm}, \mathrm{ML} \pm 0.2$ $\mathrm{mm}$ from bregma and DV $-4.1 \mathrm{~mm}$ from the dura for the RE, and AP $-1.80 \mathrm{~mm}, \mathrm{ML} \pm 1.6 \mathrm{~mm}$ from bregma and DV $-1.5 \mathrm{~mm}$ from the dura for the HP. Two screws were fixed on the skull to ensure implant stability. Optic fibers were fixed in place with dental cement (Meliodent, Heraeus).

For FluoroGold (FG) injection female mice were anaesthetized as before and $0.5 \mu \mathrm{L}$ of a $4 \%$ FG solution dissolved in saline ( $\mathrm{NaCl} 0.9 \%$ ) was unilaterally injected in the HP at the same coordinates used for the viral injection.

At the end of the surgical procedure mice were intraperitoneally (ip) injected with $1 \mathrm{ml}$ saline solution $(\mathrm{NaCl} 0.9 \%)$ and left to recover for 10-15 days. 


\section{In vivo drug injection for DREADDs experiments}

Two weeks after surgery mice underwent behavioral testing. $30 \mathrm{~min}$ before the beginning of the behavioral procedure or immediately after the end of the training phase, mice were injected with vehicle (Veh; $\mathrm{NaCl}$ $0,9 \%$ ) or CNO (1 mg/kg; Hello Bio). At the end of study phase, mice were left to rest in their waiting cages for 1 hour, after which they were put back in the resident cage. $24 \mathrm{~h}$ later mice underwent to the test phase.

\section{Light stimulation protocol for optogenetics experiments}

Four to five weeks post-AAV injection, mice underwent behavioral testing and light stimulation protocol. Immediately after the end of the study phase the implantable fibers were coupled to a $120-\mathrm{mW}, 532-$ nm diode-pumped solid-state laser (Laserlands, China) using a one-input one-output rotary joint (Thorlabs, USA) connected to a bifurcated fiber bundle (Thorlabs, USA). Power output from the tip of the bifurcated fiber bundle was about 11-12 $\mathrm{mW}$. Immediately, after the end of the study phase $30 \mathrm{~min}$ of continuous light stimulation (light-on) or no light (light off) was delivered. After photostimulation mice were left to rest for an additional $30 \mathrm{~min}$ in their waiting cages after which they were put back in the resident cage for the night. $24 \mathrm{~h}$ later mice underwent $5 \mathrm{~min}$ test phase.

\section{AAVs and FluoroGold expression verification}

At the end of the behavioral procedure for DREADDs experiment, mice were deeply anaesthetized and transcardially perfused with PBS followed by $4 \%$ paraformaldehyde (PFA; Sigma Aldrich). Brains were dissected and post-fixed for $24 \mathrm{~h}$ in PFA $4 \%$, then they were washed in PBS and put in $30 \%$ sucrose solution. $30 \mu \mathrm{m}$ coronal slices were obtained with the use of a cryostat (Leica CM3050 S) and stored in PBS and sodium azide $0.02 \%$ at $4^{\circ} \mathrm{C}$ until the beginning of the procedure. Several free-floating sections per mouse were chosen for the HP and the RE to evaluate the placement and extension of AAV injection.

For DREADDs experiments, after rinsing in 1x PBS, brain sections were incubated in blocking solution made of NGS 5\% and $0.3 \%$ Triton X-100 in PBS for 1 $\mathrm{h}$. Blocking solution was then removed and replaced with primary antibody solution made of NGS $1 \%$, Triton X-100 0.3\% and rabbit anti-HA tag (\#3724; Cell Signaling) diluted 1:500 overnight at $4^{\circ} \mathrm{C}$. Sections were washed three times in $1 \mathrm{x}$ PBS and then incubated for $2 \mathrm{~h}$ at room temperature with secondary antibody solution made with NGS 1\%, Triton X-100 0.3\% and goat-anti-rabbit Alexa Fluor 647 (Merck Millipore) diluted 1:300 in 1x PBS. After three washes with $1 \mathrm{x}$ PBS, sections were incubated for 10 mins with 4',6diamidino-2-phenylindole (DAPI), for nucleic acid staining, at room temperature and then washed three times with 1x PBS. Finally, sections were mounted on microscope glass slides and coverslipped with Mowiol (4-88; Sigma Aldrich). Control sections, which had not been exposed to primary antibody, were processed in parallel.

Slices were manually analyzed under a Nikon Ni-E fluorescence microscope equipped with Nikon DS-Ri2 camera. Only mice expressing the reporter HA in the HP or in the RE were included in the statistical analysis. For the optogenetic experiment, the brain sampling protocol was the same as that described previously for DREADDs, but in this case, HP and RE sections were only incubated for $10 \mathrm{mins}$ with DAPI and subsequently mounted on microscope glass slides and coverslipped with Mowiol.

Slices were manually analyzed under a Nikon Ni-E fluorescence microscope equipped with Nikon DS-Ri2 camera. Only mice expressing the reporter EYFP in the $\mathrm{RE}$ and the HP were included in the statistical analysis. FluoroGold (FG) injected mice were deeply anaesthetized, transcardially perfused and their brains were treated as previously described. FG labelled cells were visualized on $40 \mu \mathrm{m}$ coronal slices counterstained with a $5 \mu \mathrm{g} / \mathrm{ml}$ propidium iodide (Santa Cruz Biotechnology) staining solution in PBS $1 \mathrm{x}$ for $10 \mathrm{~min}$. Mosaic reconstruction of $\mathrm{dHP}$ and RE slices were acquired with a $6 \mathrm{x}$ objective, while RE magnification was acquired with a 10x NA 0.2 objective with a Leica Laser Microdissector.

\section{Ex vivo electrophysiological recordings in slices}

Transverse hippocampal slices (320 $\mu \mathrm{m}$ tick) were obtained using a standard protocol (Bischofberger et al., 2006) from 3 months old mice injected 4-5 weeks before with AAV-hSyn-eNpHR 3.0-EYFP in the RE. Briefly, after being anesthetized with an intraperitoneal injection of a mixture of tiletamine/zolazepam $(80 \mathrm{mg} / \mathrm{Kg})$ and xilazine $(10 \mathrm{mg} / \mathrm{Kg})$, mice were decapitated. The brain was quickly removed from the skull, placed in artificial cerebrospinal fluid (ACSF) containing (in $\mathrm{mM}$ ): sucrose $75, \mathrm{NaCl} 87, \mathrm{KCl} 2.5$, $\mathrm{NaH}_{2} \mathrm{PO}_{4} 1.25, \mathrm{MgCl}_{2} 7, \mathrm{CaCl}_{2}$ 0.5, $\mathrm{NaHCO}_{3} 25$, glucose 25. After recovery, an individual slice was transferred to a submerged recording chamber and continuously perfused at room temperature with oxygenated ACSF at a rate of $3 \mathrm{ml} / \mathrm{min}$. ASCF saturated with $95 \% \mathrm{O}_{2}$ and $5 \% \mathrm{CO}_{2}$ and contained in 
$\mathrm{mM}: \mathrm{NaCl} 125, \mathrm{KCl} 2.5, \mathrm{NaH}_{2} \mathrm{PO}_{4} 1.25, \mathrm{MgCl}_{2}$, $\mathrm{CaCl}_{2} 2, \mathrm{NaHCO}_{3} 25$, glucose 10 .

Cells were visualized with a $60 \mathrm{X}$ water immersed objective mounted on an upright microscope (Nikon, eclipse FN1) equipped with a CCD camera (Scientifica, UK). eNHpR+ fibers were photoinhibited with continuous (5-10 minutes) green light delivered through the objective and generated by a $535 \mathrm{~nm}$ LED (pE2, CoolLED, UK) under the control of the digital output of the amplifier. Whole-cell patch clamp recordings, in voltage and current clamp modes, were performed with a MultiClamp 700B amplifier (Axon Instruments, Sunnyvale, CA, USA). Patch electrodes were pulled from borosilicate glass capillaries (WPI, Florida, US); they had a resistance of 3-4 M $\Omega$ when filled with an intracellular: $\mathrm{K}$ gluconate $127, \mathrm{KCl} 6$, HEPES 10, EGTA 1, $\mathrm{MgCl}_{2} 2, \operatorname{MgATP} 4, \operatorname{MgGTP} 0.3$; the $\mathrm{pH}$ was adjusted to 7.2 with $\mathrm{KOH}$; the osmolarity was 290-300 mOsm. sEPSCs were recorded at the equilibrium potential for chloride $\left(\mathrm{E}_{\mathrm{Cl}}\right)$ that was approximately $-65 \mathrm{mV}$ based on the Nernst equation. Membrane potential values were not corrected for liquid junction potentials. The stability of the patch was checked by repetitively monitoring the input and series resistance during the experiments. Series resistance (10-20 M $\Omega$ ) was not compensated. Cells exhibiting $15 \%$ changes were excluded from the analysis.

Data were transferred to a computer hard disk after digitization with an A/D converter (Digidata 1550, Molecular Devices, Sunnyvale, CA, USA). Data acquisition (digitized at $10 \mathrm{kHz}$ and filtered at $3 \mathrm{kHz}$ ) was performed with pClamp 10.4 software (Molecular Devices, Sunnyvale, CA, USA). Input resistance and cells capacitance were measured online with the membrane test feature of the pClamp software. Spontaneous EPSCs were analyzed with pClamp 10.4 (Molecular Devices, Sunnyvale, CA, USA). This program uses a detection algorithm based on a sliding template. The template did not induce any bias in the sampling of events because it was moved along the data trace one point at a time and was optimally scaled to fit the data at each position.

\section{In vivo electrophysiological recordings from anesthetized animals}

Mice injected 4-5 weeks before with AAV-hSyneNpHR 3.0-EYFP in the RE were anesthetized with i.p. injection of a mixture of tiletamine/zolazepam (Zoletil; $80 \mathrm{mg} / \mathrm{Kg})$ and xilazine $(10 \mathrm{mg} / \mathrm{Kg})$ to induce anesthesia before surgery and during recordings. Temperature was maintained between $36-37^{\circ} \mathrm{C}$ using a feedback-controlled heating pad (FHC). A craniotomy for stimulation and recording sites was drilled between 1.6 and $2.0 \mathrm{~mm}$ posterior from bregma, and lateral coordinates were adjusted after extracellular mapping to locate the dorsal CA1 pyramidal cell layer. Light activation of eNHpR+ fibers was achieved with $50 \mathrm{~mW}$ $532 \mathrm{~nm}$ laser (SLOC Lasers, Shangai) delivered through an optical fiber $(200 \mu \mathrm{m}$ diameter, 0.22 numerical aperture NA) lowered with $10^{\circ}$ angle in the stratum radiatum. Power output at the tip of the fiber was 3-4 $\mathrm{mW}$. Continuous light was triggered for 5-10 minutes.

Extracellular recordings of local field potentials from CA1 were obtained with glass electrodes (Hingelberg, Malsfeld, Germany) prepared with a vertical puller PP830 (Narishige), and the tip was broken to obtain a resistance of 1-2 M $\Omega$. The distance between the optical fiber and the recording electrode was 150-200 $\mu \mathrm{m}$. Electrodes were filled with a standard Ringer's solution containing the following (in $\mathrm{mM}$ ): $\mathrm{NaCl} 135, \mathrm{KCl} 5.4$, HEPES 5, $\mathrm{CaCl}_{2}$ 1.8, and $\mathrm{MgCl}_{2} 1$.

Recordings were obtained with a Multiclamp 700B amplifier connected to the Digidata 1550 system. Data were acquired (digitized at $10 \mathrm{kHz}$ and filtered at 3 $\mathrm{kHz}$ ) with pClamp 10.4 software and analyzed off-line with Clampfit 10.4 (Molecular Devices).

Traces were high pass filtered $(300 \mathrm{~Hz})$ and a clampfit algorithm was used to detect extracellular spikes (MUA) setting a threshold of three standard deviations from the noise. The spontaneous MUA was estimated during 5-10 $\mathrm{min}$ before, during and after light stimulation.

\section{Western blot}

At the end of the DREADDs behavioral procedure, after one week, mice were injected again with $\mathrm{CNO}$ or Veh and, after $65 \mathrm{~min}$, they were sacrificed through cervical dislocation and their brains were collected. The dorsal hippocampi (HP) were dissected using a brain matrix (Kopf Instruments).

HP sections were homogenized with homogenizing buffer composed of $0.32 \mathrm{M}$ sucrose, $1 \mathrm{mM}$ EDTA, 1 $\mathrm{mg} / \mathrm{mL}$ BSA, 5 mM HEPES pH 7.4, 1 mM PMSF, 2 $\mathrm{mM}$ sodium orthovanadate, $10 \mathrm{mM}$ sodium fluoride, $1 \mathrm{x}$ Sigma protease inhibitor cocktail in distilled $\mathrm{H}_{2} \mathrm{O}$. Protein concentration was determined by Bradford assay (Bio-Rad).

Dorsal HP homogenates aliquots $(20 \mu \mathrm{g} / \mathrm{lane})$ from $\mathrm{CNO}$ and saline treated mice were first boiled at $95^{\circ} \mathrm{C}$ for $5 \mathrm{~min}$ and then separated on $10 \%$ polyacrylamide gel and transferred on PVDF membranes (Immobilon$\mathrm{P}$ transfer membrane; Merck Millipore). Membranes were then blocked with $5 \%$ non-fat powdered milk in 
TBS $+0.01 \%$ Tween-20 (TBS-T) for $1 \mathrm{~h}$ at room temperature followed by overnight incubation at $4{ }^{\circ} \mathrm{C}$ with rabbit anti-glutamate receptor 1 (AMPA subtype) phosphoSer 845 antibody (1:500; ab76321, Abcam) and rabbit anti-glutamate receptor 1 (AMPA subtype) antibody (1:500; ab31232, Abcam).

Subsequently, blots were washed with TBS-T and secondary antibodies (1:5000; Bio-Rad) were applied for $1 \mathrm{~h}$ at room temperature. Bands were detected by chemiluminescence and quantified using Image software (NIH, Bethsda, MD). Data are expressed as \% from Veh treated samples.

\section{c-Fos counting and GAD67 co-localization by immunofluorescence}

After one week from the behavioral evaluation, mice from the RE-HP optogenetic inhibition experiment were exposed to the habituation and the study phase of the 6-DOT (high memory load) followed by $30 / 30 \mathrm{~min}$ of light on/off stimulation or $60 \mathrm{~min}$ light off as previously done. Mice were then deeply anaesthetized and transcardially perfused with 1x PBS followed by 4\% paraformaldehyde (PFA; Sigma Aldrich). Brains were collected and post-fixed for $24 \mathrm{~h}$ in PFA and passed in a $30 \%$ sucrose solution. $30 \mu \mathrm{m}$ coronal slices were obtained with the use of a vibratome (Leica VT1000 S) and stored in PBS and sodium azide $(0.02 \%)$ at $4^{\circ} \mathrm{C}$ until histological processing. Slices were incubated with primary antibodies against: c-Fos (1:1000, 226 017, Synaptic System) and GAD67 (1:700, MAB5406, Merck Millipore) after a blocking step with PBS-Triton X-100 0.3\% and 5\% normal goat serum (NGS). Slices were subsequently washed with PBS and incubated for $1 \mathrm{~h}$ with the proper secondary antibodies $\left(1: 400\right.$, goat anti-rat Alexa-Fluor ${ }^{\circledR}$ 568, ab175476, abcam; 1:400, goat anti-mouse AlexaFluor ${ }^{\circledR} 488$, AP124JA4, Merck Millipore). For nuclear counterstaining slices were incubated with DAPI (1:1000, $10 \mathrm{~min}, \mathrm{D} 1306$, Invitrogen) before mounting and inclusion with a Mowiol 4-88 (Sigma Aldrich) solution.

Images of c-Fos and GAD67 co-localization were acquired as z-stacks ( 9 sections every $2 \mu \mathrm{m}$ ) using a spinning disk confocal microscope (X-light V3 Crest Optics) using a 25x NA 1.05 silicon oil immersion objective. All images were acquired using a Prime BSI with 2048 x 2048 pixels. For all the experiments 3-5 slice per mouse and 3-4 mice per group were acquired and analyzed using QuPath 0.2.3 (Bankhead et al., 2017). For each slice three alternated z-stack were sampled with a region of interest (ROI) in the pyramidal layer (py) and in the stratum oriens (so) of the CA1. c-
$\mathrm{Fos}^{+}$cells were first counted in the py and the so independently with the cell detection tool by QuPath. Subsequently, the c-Fos ${ }^{+}$cells of the so were classified as $\mathrm{GAD}^{+}$or $\mathrm{GAD}^{-}$using the single measurement classifier tool by QuPath. c-Fos ${ }^{+}$and c-Fos ${ }^{+} / \mathrm{GAD}^{+}$cells were then summed per area per ROI for each HP side and averaged per slice. The percentage of $\mathrm{c}-\mathrm{Fos}^{+} / \mathrm{GAD}^{+}$ cells was calculated for the total number of c-Fos ${ }^{+}$cells. Slices were averaged per groups (light off $v s$ light on) for female and male mice.

p-EIF2 $\alpha /$ EIF $2 \alpha$ quantification in excitatory neurons One week following the behavioral evaluation, mice from the RE-HP optogenetic inhibition experiment were exposed to the habituation and study phase of the 6-DOT (high memory load) followed by $30 / 30 \mathrm{~min}$ of light on/off stimulation or $60 \mathrm{~min}$ light off as previously done. Mice were then deeply anaesthetized and transcardially perfused with 1x PBS followed by $4 \%$ paraformaldehyde (PFA; Sigma Aldrich). Brains were collected and post-fixed for $24 \mathrm{~h}$ in PFA and passed in a $30 \%$ sucrose solution. $30 \mu \mathrm{m}$ coronal slices were obtained with the use of a vibratome (Leica VT1000 S) and stored in PBS and sodium azide $(0.02 \%)$ at $4{ }^{\circ} \mathrm{C}$ until histological processing. Slices were incubated with primary antibodies against: p-EIF2 $\alpha$ (Ser51) (1:200, 3597, Cell Signaling) or EIF2 $\alpha(1: 800,5324$, Cell Signaling), and GAD67 (1:700, MAB5406, Merck Millipore) and NeuN (1:500, ABN90, Merck Millipore) after a blocking step with PBS-Triton X-100 0.3\% and $5 \%$ normal goat serum (NGS). Slices were subsequently washed with PBS and incubated for $2 \mathrm{~h}$ with the proper secondary antibodies $(1: 300$, goat antirabbit Alexa-Fluor ${ }^{\circledR}$ 647, AP187SA6, Merck Millipore; 1:300, goat anti-guinea pig Alexa-Fluor ${ }^{\circledR}$ 568, ab175714, Abcam; 1:400, goat anti-mouse AlexaFluor® 488, AP124JA4, Merck Millipore). For nuclear counterstaining slices were incubated with DAPI (1:1000, $10 \mathrm{~min}, \mathrm{D} 1306$, Invitrogen) before mounting and inclusion with Mowiol 4-88 (Sigma Aldrich) solution.

Images of p-EIF2 $\alpha / \mathrm{GAD} 67 / \mathrm{NeuN}$ and EIF2 $\alpha /$ GAD67/NeuN were acquired using a confocal microscope (Leica SP5) with a 40x objective and a $1.5 \mathrm{x}$ digital zoom. All images were acquired with a $1024 \times 1024$ pixel resolution. For all the experiments 3 slice per mouse and 3 mice per group were acquired and analyzed using ImageJ (NIH). Each image was sampled in the GAD67 $7^{+}$channel with 4 regions of interest (ROIs) positioned on the excitatory neurons, using the pattern of GAD67 cytoplasmatic expression as a discriminatory marker between excitatory neurons 
(GAD67-). NeuN was used as a counterstaining to recognize neurons. The fluorescence intensity (AU) of p-EIF $2 \alpha$ or EIF $2 \alpha$ was measured in each ROI and averaged for category of excitatory neurons (GAD67 $7^{-}$ per groups (light off $v s$ light on) for female and male mice. The number of p-EIF $2 \alpha^{+}$spots was correlated to the fluorescence intensity values per ROI for a subgroup of slice from males and females to prove the reliability of the fluorescence intensity signal as an index of the amount of p-EIF2 $\alpha$ levels (Extended Data Fig. 6c). Fluorescence intensity was averaged per slices of each experimental condition.

\section{Statistics}

Discrimination index at T3 (test phase) was analyzed with a one-way or a three-way ANOVA. Depending on the experiment, sex (two levels: male, female), load (two levels:6-IOT, 6-DOT), delay (two levels: $1 \mathrm{~min}$, $24 \mathrm{~h}$ ) and treatment (two levels: Veh, CNO for DREADDs experiments, or light on, light off for optogenetics experiments) were used as between factors.

For single object comparisons, we used a one-way ANOVA for repeated measures with objects (six levels: New, F1-F5) as repeated measures, followed by Dunnett post-hoc analysis.

Counting of c-Fos ${ }^{+}$cells per slice was averaged per animal and, for each brain region, data were normalized on naïve average considering the antero-posterior extent, thus values were expressed as $\%$ on naïve expression. A two-way ANOVA for sex (two levels: male, female) and test (two levels: naïve, test) was used. Bonferroni post-hoc analysis was carried out when the $\mathrm{p}$-value for interaction of sex $\mathrm{x}$ test was significant; if this was not the case a one-way ANOVA for sex or treatment separately was used.

For Western Blot analysis, a one-way ANOVA for treatment (two levels: saline, $\mathrm{CNO}$ ) was used to analyze data for GluR1 p-S845 and data, normalized on GluR1 levels, are expressed as \% vs 3 months treated samples. For MUA analysis Wilcoxon matched-pairs signed rank test was used to assess $\mathrm{CNO}$ effect on firing rate.

Data availability statement: All data is available upon reasonable request to the corresponding author.

\section{Methods references}

35. Byers, S. L., Wiles, M. V, Dunn, S. L. \& Taft, R. A. Mouse Estrous Cycle Identification Tool and Images. 7, 2-6 (2012).
36. Ainge, J. A. et al. The role of the hippocampus in object recognition in rats : Examination of the influence of task parameters and lesion size. Behav. Brain Res. 167, 183-195 (2006).

37. Guzowski, J. F., Setlow, B., Wagner, E. K. \& McGaugh, J. L. Experience-dependent gene expression in the rat hippocampus after spatial learning: a comparison of the immediate-early genes Arc, c-fos, and zif268. J. Neurosci. 21, 5089-5098 (2001).

38. Maviel, T., Durkin, T. P., Menzaghi, F. \& Bontempi, B. Sites of Neocortical Reorganization Critical for Remote Spatial Memory. Science (80-. ). 305, 96-99 (2004).

39. De Leonibus, E., Mele, A., Oliverio, A. \& Pert, A. Distinct pattern of c-Fos mRNA expression after systemic and intra-accumbens amphetamine and MK-801. Neuroscience 115, 67-78 (2002). 